\title{
Clinical role of a subcortical communication
}

\author{
Esther A. Pelzer ${ }^{1,2 *}$, Lars Timmermann ${ }^{2}$, Marc Tittgemeyer ${ }^{1}$
}

'Translational Neurocirciutry Group, Max-Planck Institute for Metabolism Research Cologne, Cologne, Germany

${ }^{2}$ Department of Neurology, University Clinics Cologne, Cologne, Germany

Article Info

\section{Article Notes}

Received: June 06, 2016

Accepted: August 08, 2016

\section{*Correspondence:}

Dr. Esther Pelzer

Translational Neurocircuitry Group, Max-Planck-Institute for

Metabolism Research

50931 Cologne, Germany

E-mail: esther.pelzer@sf.mpg.de

() 2016 Pelzer EA. This article is distributed under the terms of the Creative Commons Attribution 4.0 International License

\section{Keywords}

Cerebellum

Basal Ganglia

Thalamus

Axon degeneration

Oscillatory activity

\section{ABSTRACT}

Subcortical communication is an important underlying feature for the smooth performance of motor behaviour. Especially movement disorders like Parkinson's disease show impairment in the basal-ganglio-thalamic and cerebello-thalamic communication; but also an impairment of the direct communication between these two structures has been proposed.

In this review we highlight important clinical findings concerning the pathological communication between these subcortical structures; additionally we propose a new hypothesis in the development of neurodegenerative disease: we assume that axon degeneration is crucially implicated in the development of parkinsonian symptoms and link the current findings to the development of pathological oscillatory activity. New techniques like probabilistic tractography now offer the possibility to in vivo measure axon degeneration by the determination of connectivity decline and allow the combination with electrophysiological recording. We hypothesize that a change in frequency bands in oscillatory activity might be a product of underlying axonal degeneration; moreover axonal degeneration might be worsened by pathological oscillatory activity resulting in a vicious circle. The thalamus, as main relay station between the basal ganglia and the cerebellum seems to be involved in this disease pathology in Parkinson's disease.

\section{Introduction}

The thalamus is the important relay station of cortical and subcortical regions. It integrates information of different neuronal circuits, in particular between the basal ganglia (BG) and the cerebellum. Whereas cerebellar projections rather target posterior areas, BG projections are more located in anterior parts of the thalamus, specifically in the motor thalamus ${ }^{1}$. The organisation of the BG system and its projections is segregated with functionally organized loops ${ }^{2}$, with the supplementary motor area (SMA) as one of the main receiving regions ${ }^{3}$. Next to pure segregated information processing also overlapping regions exist, which might facilitate the informational exchange between basal ganglia and cerebellum ${ }^{1,4}$. The idea of such overlapping regions derived from a recent study, where a direct di- and trisynaptical exchange between the cerebellum and the BG has been found in the monkey ${ }^{5,6}$; the existence of such an interconnection has also been recently reported in humans ${ }^{7}$; the existence of such overlapping regions has, however, been controversially discussed in literature before ${ }^{8}$. In this review we want to highlight their relevance in the development of movement disorders like Parkinson's disease (PD). 


\section{Clinical Manifestation of Altered BG and Cerebellar} Circuitry

According to the SMA being a critical component in effectively initiating (particularly internally) generated movements ${ }^{9}$, BG-thalamo-cortical pathway disruptions secondary to dopamine depletion might offer an explanation of the development of bradykinesia in PD. Thus, decreased activity in the SMA is postulated as consequence of insufficient BG-thalamo-cortical facilitation in $\mathrm{PD}^{2}$. But this mechanism might not be exclusively traceable in PD. Decrease of functional connectivity in the BGthalamo-motor loop along with increased impairment of self-initiated movements has been demonstrated during physiological aging ${ }^{10}$. At the same time, studies indicate that with increasing deficiency in the BG-thalamo-cortical circuit, an apparent compensatory effect in the cerebellothalamo-cortical loop gains in importance during the course of PD. Furthermore, the role of the cerebellum in the development of parkinsonian symptoms like bradykinesia and rigidity has been highlighted recently in addition to BG-output insufficiency ${ }^{11}$. This recruitment of the cerebello-thalamo-cortical circuit increases concomitantly with PD progression ${ }^{12}$. Whereas PD patients remain able to sustain externally triggered movements thereby compensating the deficiency of self-initiated movements with externally triggered tasks in initial stages of their disease, this compensation becomes increasingly impaired during disease progression ${ }^{13}$. Cerebellar overactivation is corroborated in patients suffering from PD in a substantial amount of functional neuroimaging studies using PET or blood oxygen level-dependent functional MRI: (a) during externally or internally timed simple finger movements ${ }^{14-16}$, (b) motor timing paradigms ${ }^{9}$, (c) complex sequential movements ${ }^{17}$, (d) bimanual twohand coordinated tasks ${ }^{18}$ or when (e) performing two different motor tasks simultaneously ${ }^{19}$. According to methodological limitations of in vivo measurements, the underlying structural pathology of this compensatory function in PD has never been systematically analysed so far. Probabilistic tractography might offer a new way to indirectly quantify neuronal count by connectivity decline and in vivo determine the degree of overlap in different brain regions ${ }^{20}$. The complex interplay between $\mathrm{BG}$ and cerebellum at thalamic level might be facilitated due to overlapping areas to enhance information processing.

\section{Homöostasis of BG-cerebellar System and Firing} Rates

The integration of BG and cerebellar projections within the thalamus is an important underlying feature for smooth movement sequences. In animal studies it has been discussed that an inactivation of the sensorimotor territory of the globus pallidus internus (GPi, the main BG motor output) results in an impaired execution of movements ${ }^{21}$.
Similarly, affection of movement execution is evident during aging as well, resulting in both slow and more variable execution of movements ${ }^{22}$. In PD, comparable motor alterations, clinically reflected as bradykinesia, reductions of blink rate or reduced automated movements are almost pathognomonic even in early stages and often instigate patients to attend for medical advice. In later PD stages, patients' quality of life is massively constrained due to the difficulties initiating movements as well as performing complex movements ${ }^{23}$. This said, it again offers room for speculation about similarities between physiologic ageing and PD; at the same time the question arises what yields to an altered BG-output.

Classically, the alteration of the $B G$ output to the thalamus is explained by an excessive inhibition of external globus pallidus (GPe) within the indirect pathway. This evokes a disinhibition of the subthalamic nucleus (STN), which in turn provides excessive excitatory drive to the basal ganglia output nuclei GPi / substantia nigra (pars reticulata; $\mathrm{SNr}$ ). Due to an excessive thalamic inhibition a reduced inhibitory input to $\mathrm{GPi} / \mathrm{SNr}$ through the direct pathway is reinforced. This is reinforced by reduced inhibitory input to $\mathrm{GPi} / \mathrm{SNr}$ through the direct pathway. Overall, these effects are postulated to result in a reduction in the usual reinforcing influence of the motor circuit upon cortically initiated movements ${ }^{2,24}$. Next to this classical view, however, experimental findings testing this model have given inconsistent results and several predictions of the classical BG model have not been validated $^{25}$. Consecutively, alternative pathophysiological mechanisms have been proposed including increased oscillatory activity - particularly in the beta frequency band -, increased synchronization of neuronal activity, or increased bursting ${ }^{26}$.

\section{Axon Degeneration in Movement Disorders}

A possible pathomechanism resulting in alterations of electrical activity of BG neurons in PD proposes a dying-back mechanism originating in striatal terminals ${ }^{27}$. The so-called axon degeneration might rather account for degeneration of the BG system than an affection of the soma, as previously assumed ${ }^{28-31}$. Recent evidence suggests axonal damage being a general principle in various neurodegenerative disorders such as amyotrophic lateral sclerosis (ALS) or $\mathrm{PD}^{32}$. There is not much literature to the changes in PD. But there is a well described model of neuronal vulnerability in $\mathrm{ALS}^{33}$ , demonstrating local imbalances in energy homeostasis amplifying neurodegeneration, while at the same time yielding an altered signalling of the affected neurons. Gradually lowering the ATP steady state concentration, the authors report no changes in spontaneous firing. Interestingly, however, lowered ATP concentrations resulted in an imbalance of homeostasis of the cell when higher frequency firing was imposed. It was concluded 
that motor neuron instability results from increasing firing rates and decreasing ATP production for its compensation. Additionally, the energetic imbalance is aggravated with rising Calcium concentrations due to mitochondrial overload, emerging again in reduced ATP synthesis. Finally, with an ATP deficit imposed upon the axonal end, local influx of high-concentration ions appears to spill the metabolic deficit into neighbouring sections. The local energy crisis thereby propagates to neighbouring compartments, eventually spreading from the distal axon to the soma ${ }^{34}$ . These processes illustrate how even small local energy dysfunction may grow to cell-wide critical proportions in neurons with a vulnerable relationship between neuronal morphology, neuronal firing, and metabolic load.

Interestingly, an involvement of voltage-gated calcium channels and ATP-sensitive potassium channels have been discussed to be present in neurodegenerative disorders like PD; here especially dopaminergic midbrain neurons of the substantia nigra seem to be specifically vulnerable yielding to a selective degeneration ${ }^{35}$. But also in other regions of the $\mathrm{BG}$, like the $\mathrm{GPi}$, parvalbumin (PV) concentrations, a $\mathrm{Ca}^{2+}$-binding protein, are markedly reduced in $\mathrm{PD}^{36,37}$. Although the mean estimates for GPi volume and the numbers of GPi neurons in PD were not significantly different from those for the controls in an earlier study, sub-population of neurons (PV positive neurons) that project from the GPi to the thalamus were selectively and significantly decreased ${ }^{38}$. Past studies suggest, that PV plays an important role for repetitive fast-firing ${ }^{39}$; herewith it appears, that subpopulations of GPi have altered firing capacity in advanced PD patients. This has been expressed by an excessive synchronicity of neurons in the GPi found during microelectrode recordings with an impairment of motor processing reflected by a bradykinesia ${ }^{40}$. Here, on a cellular level, pallidal gap junctions, that are strongly involved in the ion exchange between the extra- and intracellular space could play a major role modifying this synchrony, because they show functional plasticity under the influence of dopamine and after neuronal injury ${ }^{41}$.

\section{Discussion}

Intentional movements rely on a closely interrelated brain network including several regions. In essence, movements initiate in the cerebral motor cortex projecting directly - but also indirectly via local premotor circuits - to the brain stem or spinal motor neurons that again convey movement information to the muscles. Before a motor signal descends from the motor cortex to the brain stem and spinal cord, however, several cortical and subcortical centers, including the basal ganglia and the cerebellum, pose their influence upon the motor cortex 'shaping' the final, descending signal. Whereas the prevalent functional role of the basal ganglia lies in the learning and selection appropriate motor or behavioural programs ${ }^{42}$, specifically via reinforcement learning; the cerebellum mainly takes the supervised learning of motor control into account ${ }^{43}$ . In addition to integrating already learned information into motor control, both systems show involvement in the integration of non-motor behaviour such as e.g., limbic and/or associative loops ${ }^{44,45}$. This illustrates the complex information processing within ancient subcortical structures taking place next to cortical processing. With the appearance of neocortex in mammals, BG outputs to motor cortex via thalamus became of greater significance, especially in primates, in which a parallel expansion of cerebral cortex and BG occurred ${ }^{46}$. Despite the general evolutionary trend for neocortex and cerebellum to change in tandem, humans and other apes have a significantly larger cerebellum in relation to neocortex size than other anthropoid primates ${ }^{47}$. Thus, it illustrates the need for careful analyses of the interaction of the cerebellum, the BG and the cortex despite the massive development of the neocortex.

In this review we tried to expand the actual view of BG-cerebellar interaction with the thalamus to a complex integration system, in which we have overlapping regions next to a pure segregated informational processing. Together with the cerebral cortex these regions regulate complex motor paradigms and might even be part of a compensatory function in movement disorders like PD. We hypothesize that a change in frequency bands in oscillatory activity might be a product of underlying axonal degeneration and that axonal degeneration can also be worsened by pathological oscillatory activity resulting in a vicious circle. The thalamus, as main relay station between the basal ganglia and the cerebellum seems to be involved in this disease pathology in Parkinson's disease. In future, the combination of in vivo tractography and electrophysiological mapping might help to solve the question of the development of degenerative movement disorders like PD.

\section{Conflicts of interest}

LT received payments as a consultant for Medtronic Inc, Boston Scientific, SAPIENS, St. Jude Medical, Bayer Healthcare, UCB Schwarz Pharma, Archimedes Pharma. L.T. received honoraria as a speaker on symposia sponsored by TEVA Pharma, Lundbeck Pharma, Bracco, Gianni PR, Medas Pharma, UCB Schwarz Pharma, Desitin Pharma, Boehringer Ingelheim, GlaxoSmithKline, Eumecom, Orion Pharma, Medtronic, Boston Scientific, Cephalon, Abott, GE Medical, Archimedes, Bayer, TAD Pharma.

\section{Acknowledgements}

E.A.P, L.T., and M.T. were supported by funding of the German Research Foundation in the Clinical Research Group 219. M.T. is also supported by funding of the German Research Foundation in the transregional Collaborative Research Centre 134. 


\section{References}

1. Pelzer EA, Melzer C, Timmermann L, Von cramon DY, Tittgemeyer M. Basal ganglia and cerebellar interconnectivity within the human thalamus. Brain Struct Funct. 2016;

2. Delong MR. Primate models of movement disorders of basal ganglia origin. Trends Neurosci. 1990;13(7):281-5.

3. Schell GR, Strick PL. The origin of thalamic inputs to the arcuate premotor and supplementary motor areas. J Neurosci. 1984;4(2):53960.

4. Sakai ST, Inase M, Tanji J. Comparison of cerebellothalamic and pallidothalamic projections in the monkey (Macaca fuscata): a double anterograde labeling study. J Comp Neurol. 1996;368(2):215-28.

5. Hoshi E, Tremblay L, Féger J, Carras PL, Strick PL. The cerebellum communicates with the basal ganglia. Nat Neurosci. 2005;8(11):1491-3.

6. Bostan AC, Dum RP, Strick PL. The basal ganglia communicate with the cerebellum. Proc Natl Acad Sci USA. 2010;107(18):8452-6.

7. Pelzer EA, Hintzen A, Goldau M, Von cramon DY, Timmermann L, Tittgemeyer M. Cerebellar networks with basal ganglia: feasibility for tracking cerebello-pallidal and subthalamo-cerebellar projections in the human brain. Eur J Neurosci. 2013;38(8):3106-14.

8. Ilinsky IA, Kultas-ilinsky K. An autoradiographic study of topographical relationships between pallidal and cerebellar projections to the cat thalamus. Exp Brain Res. 1984;54(1):95-106.

9. Jahanshahi M, Jenkins IH, Brown RG, Marsden CD, Passingham RE, Brooks DJ. Self-initiated versus externally triggered movements I. An investigation using measurement of regional cerebral blood flow with PET and movement-related potentials in normal and Parkinson's disease subjects. Brain. 1995;118(4):913-933.

10. Taniwaki T, Okayama A, Yoshiura T, Togao O, Nakamura Y, Yamasaki $\mathrm{T}$, et al. Functional network of the basal ganglia and cerebellar motor loops in vivo: different activation patterns between self-initiated and externally triggered movements. Neuroimage. 2006;31(2):745-53.

11. Wu T, Hallett M. The cerebellum in Parkinson's disease. Brain. 2013; 136: 696-709.

12. Sen S, Kawaguchi A, Truong Y, Lewis MM, Huang X. Dynamic changes in cerebello-thalamo-cortical motor circuitry during progression of Parkinson's disease. Neuroscience. 2010;166(2):712-9.

13. Taniwaki T, Yoshiura T, Ogata K, Togao O, Yamashita K, Kida H, et al. Disrupted connectivity of motor loops in Parkinson's disease during self-initiated but not externally-triggered movements. Brain Research 2013; 1512: 45-59.

14. Rascol O, Sabatini U, Fabre N, et al. The ipsilateral cerebellar hemisphere is overactive during hand movements in akinetic parkinsonian patients. Brain. 1997;120( Pt 1):103-10.

15. Wu T, Hallett M. A functional MRI study of automatic movements in patients with Parkinson's disease. Brain. 2005;128(Pt 10):2250-9.

16. Cerasa A, Hagberg GE, Peppe A, et al. Functional changes in the activity of cerebellum and frontostriatal regions during externally and internally timed movement in Parkinson's disease. Brain Res Bull. 2006;71(1-3):259-69.

17. Catalan MJ, Ishii K, Honda M, Samii A, Hallett M. A PET study of sequential finger movements of varying length in patients with Parkinson's disease. Brain. 1999;122 ( Pt 3):483-95.

18. Wu T, Wang L, Hallett M, Li K, Chan P. Neural correlates of bimanual anti-phase and in-phase movements in Parkinson's disease. Brain. 2010;133(Pt 8):2394-409.

19. $\mathrm{Wu} \mathrm{T}$, Hallett $\mathrm{M}$. Neural correlates of dual task performance in patients with Parkinson's disease. J Neurol Neurosurg Psychiatr. 2008;79(7):760-6
20. Jbabdi S, Sotiropoulos SN, Haber SN, Van essen DC, Behrens TE. Measuring macroscopic brain connections in vivo. Nat Neurosci. 2015;18(11):1546-55.

21. Desmurget M, Turner RS. Motor sequences and the basal ganglia: kinematics, not habits. J. Neurosci. 2010; 30: 7685-7690.

22. Ketcham CJ, Stelmach GE. Movement control in the older adult. Technology for adaptive aging. 2004; 64-92.

23. Chapuis S, Ouchchane L, Metz O, Gerbaud L, Durif F. Impact of the motor complications of Parkinson's disease on the quality of life. Mov. Disord. 2005; 20:224-230.

24. Albin RL, Young AB, Penney JB. The functional anatomy of disorders of the basal ganglia. Trends Neurosci. 1995;18(2):63-4.

25. Galvan A, Wichmann T. Pathophysiology of parkinsonism. Clin Neurophysiol. 2008; 119:1459-1474.

26. Lobb C. Abnormal Bursting as a Pathophysiological Mechanism in Parkinson's Disease. Basal Ganglia. 2014;3(4):187-195.

27. Hornykiewicz 0. Biochemical aspects of Parkinson's disease. Neurology. 1998;51(2 Suppl 2):S2-9.

28. Cheng HC, Ulane CM, Burke RE. Clinical progression in Parkinson disease and the neurobiology of axons. Ann Neurol. 2010;67(6):71525 .

29. Burke RE, O'malley K. Axon degeneration in Parkinson's disease. Exp Neurol. 2013;246:72-83.

30. O'malley KL. The role of axonopathy in Parkinson's disease. Exp Neurobiol. 2010;19(3):115-9.

31. Tagliaferro P, Burke RE. Retrograde Axonal Degeneration in Parkinson Disease. J Parkinsons Dis. 2016;6(1):1-15.

32. Conforti L, Gilley J, Coleman MP. Wallerian degeneration: an emerging axon death pathway linking injury and disease. Nat Rev Neurosci. 2014;15(6):394-409.

33. Le masson G, Przedborski S, Abbott LF. A computational model of motor neuron degeneration. Neuron. 2014;83(4):975-88.

34. Roselli F, Caroni P. Modeling neuronal vulnerability in ALS. Neuron 2014;83(4):758-60.

35. Cataldi M. The changing landscape of voltage-gated calcium channels in neurovascular disorders and in neurodegenerative diseases. Curr Neuropharmacol. 2013;11(3):276-97.

36. Lucas EK, Markwardt SJ, Gupta S, et al. Parvalbumin deficiency and GABAergic dysfunction in mice lacking PGC-1alpha. J Neurosci. 2010;30(21):7227-35.

37. Dragicevic E, Schiemann J, Liss B. Dopamine midbrain neurons in health and Parkinson's disease: emerging roles of voltagegated calcium channels and ATP-sensitive potassium channels. Neuroscience. 2015;284:798-814.

38. Hardman CD, Halliday GM. The internal globus pallidus is affected in progressive supranuclear palsy and Parkinson's disease. Exp Neurol. 1999;158(1):135-42

39. Celio MR. Calbindin D-28k and parvalbumin in the rat nervous system. Neuroscience. 1990;35(2):375-475.

40. Hammond C, Bergman H, Brown P. Pathological synchronization in Parkinson's disease: networks, models and treatments. Trends Neurosci. 2007;30(7):357-64.

41. Schwab BC, Heida T, Zhao Y, Van gils SA, Van wezel RJ. Pallidal gap junctions-triggers of synchrony in Parkinson's disease?. Mov Disord. 2014;29(12):1486-94. 
42. Groenewegen HJ. The Basal Ganglia and Motor Control. Neural Plasticity. 2003; 10:107-120.

43. Doya K. Complementary roles of basal ganglia and cerebellum in learning and motor control. Curr. Opin. Neurobiol. 2000; 10:732739.

44. Middleton FA, Strick PL. Basal ganglia and cerebellar loops: motor and cognitive circuits. Brain Res Brain Res Rev. 2000;31(2-3):236-50.
45. Redgrave P, Rodriguez M, Smith Y, et al. Goal-directed and habitual control in the basal ganglia: implications for Parkinson's disease. Nat Rev Neurosci. 2010;11(11):760-72.

46. Reiner A. You cannot have a vertebrate brain without a basal ganglia. The Basal Ganglia IX, 2009; pp:3-24.

47. Barton RA, Venditti C. Rapid evolution of the cerebellum in humans and other great apes. Curr Biol. 2014;24(20):2440-4. 